\title{
Heat capacity anomaly near the lower critical consolute point of triethylamine-water
}

\author{
Anne C. Flewelling, a) Rohan J. DeFonseka, ${ }^{\text {b) }}$ Nikfar Khaleeli, ${ }^{\text {c) }}$ J. Partee, ${ }^{\text {d) }}$ \\ and D. T. Jacobs ${ }^{\Theta 1}$ \\ Physics Department, The College of Wooster, Wooster, Ohio 44691
}

(Received 5 December 1995; accepted 20 February 1996)

\begin{abstract}
The heat capacity of the binary liquid mixture triethylamine-water has been measured near its lower critical consolute point using a scanning, adiabatic calorimeter. Two data runs are analyzed to provide heat capacity and enthalpy data that are fitted by equations with background terms and a critical term that includes correction to scaling. The critical exponent $\alpha$ was determined to be $0.107 \pm 0.006$, consistent with theoretical predictions. When $\alpha$ was fixed at 0.11 to determine various amplitudes consistently, our values of $A^{+}$and $A^{-}$agreed with a previous heat capacity measurement, but the value of $A^{+}$was inconsistent with values determined by density or refractive index measurements. While our value for the amplitude ratio $A^{+} / A^{-}=0.56 \pm 0.02$ was consistent with other recent experimental determinations in binary liquid mixtures, it was slightly larger than either theoretical predictions or recent experimental values in liquid-vapor systems. The correction to scaling amplitude ratio $D^{+} / D^{-}=0.5 \pm 0.1$ was half of that predicted. As a result of several more precise theoretical calculations and experimental determinations, the two-scale-factor universality ratio $X$, which we found to be $0.019 \pm 0.003$, now is consistent among experiments and theories. A new "universal" amplitude ratio $R_{B}^{ \pm}$involving the amplitudes for the specific heat was tested. Our determination of $R_{B_{\mathrm{cr}}}^{+}=-0.5 \pm 0.1$ and $R_{B_{\mathrm{cr}}}^{-}=-1.1 \pm 0.1$ is smaller in magnitude than predicted and is the first such determination in a binary fluid mixture. (C) 1996 American Institute of Physics. [S0021-9606(96)50820-2]
\end{abstract}

\section{INTRODUCTION}

Critical point phenomena have been studied over a long period of time with substantial recent effort toward modeling complex systems both near a critical point and over an extended region in thermodynamic space. Large density or concentration fluctuations near a system's critical point effectively mask the identity of the system and produce universal phenomena which have been well studied in simple liquidvapor and liquid-liquid systems. ${ }^{1-3}$ Such systems have provided useful model systems to test theoretical predictions which can then be extended to more complicated systems. Along various thermodynamic paths, several quantities exhibit a simple power-law dependence close to the critical point. The critical exponents describing these relationships are universal and should depend only on a universality class determined by the order-parameter and spatial dimensionality of the system. Binary fluid mixtures, liquid-vapor systems, and uniaxial ferromagnetic materials are thought ${ }^{1-3}$ to belong to the three dimensional, Ising model which, in a renormalization group (RG) context, predicts ${ }^{4}$ values, for the

\footnotetext{
a'Present address: Department of Biophysics, University of Rochester, Rochester, NY 14627

h)Present address: Department of Electrical Engineering, SUNY at Binghamton, Binghamton, NY 13902.

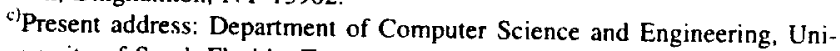
versity of South Florida, Tampa. FL 33620.

${ }^{d)}$ Present address: Department of Physics, Iowa State University, Ames, IA 50011.

e'Author to whom correspondence should be addressed.
}

critical exponents. These values, for the most part, agree very well with experimental results. ${ }^{1-3}$ Several universal amplitude ratios have also been predicted and many have also been experimentally tested with mixed results. ${ }^{5}$ A particularly powerful observation is that only two critical exponents are linearly independent, and that the leading amplitudes are interrelated using only two scale factors. ${ }^{1-3}$ Thus the universality of the exponents could be used with two experiments to determine all the leading critical behavior of a given system.

The heat capacity provides a delicate probe of the system near a critical point and can determine essential amplitude and exponent values. In particular, a precise measurement of the heat capacity of the binary fluid mixture triethylamine and water will help settle a dispute in the literature about the amplitude in the one-phase region and its effect on the universal ratio $X$, measure the universal ratio of the amplitudes above and below the critical temperature, and give the first attempt in binary liquid mixtures at using a new "universal" amplitude relation $R_{B_{\mathrm{cr}}}^{ \pm}$to reduce the number of fitting parameters while testing theoretical predictions.

The heat capacity has a weak divergence near the critical point that is governed by the critical exponent $\alpha$, has a critical contribution $B_{c}$ to the background heat capacity, and has correction to scaling terms that extend the theoretical description further from the critical point. ${ }^{3.6}$ The critical point influences the system properties over a global region: ${ }^{7.8}$ however, this experiment measures behavior near the critical point and we will use the simpler formalism that is appropri- 
ate there. While a vapor-liquid system measured along the critical isochore would have a weak divergence in the heat capacity at constant volume, a binary fluid mixture near its critical consolute point will have the same type of divergence in its heat capacity at constant pressure when measured along a path of constant, critical composition $x=x_{c} ;$

$$
\begin{aligned}
C_{p x}= & \left(B_{b g}+B_{c}\right)+E t+\frac{A^{+}}{\alpha}|t|^{-\alpha}\left(1+D^{+}|t|^{\Delta_{1}}\right. \\
& +\cdots) \text { one phase, } \\
C_{p x}= & \left(B_{b g}+B_{c}\right)+E t+\frac{A^{-}}{\alpha}|t|^{-\alpha}\left(1+D^{-}|t|^{\Delta_{1}}\right. \\
& +\cdots) \text { two phase, }
\end{aligned}
$$

where $C_{p x}$ is the temperature dependent heat capacity at constant pressure and at the critical composition, $B_{b g}$ is the background heat capacity far from the critical temperature $T_{c}$ in the one-phase region, $t=\left(T-T_{c}\right) / T_{c}$ is the reduced temperature, $A^{+1-}$ is the amplitude of the leading divergence, $D^{+/-}$is the amplitude of the first correction to scaling term, and $\Delta_{1}$ is the universal exponent for the correction term. The one and two phase amplitudes are denoted by the + and superscript respectively; the background terms and the critical exponents are predicted ${ }^{6}$ to be the same above and below the critical point. A linear background term $E t$ arises from the regular part of the free energy and hence should be the same above and below the critical point. The critical exponents $\alpha$ and $\Delta_{1}$ are predicted ${ }^{9}$ to be $0.110 \pm 0.003$ and 0.51 \pm 0.03 , respectively.

The critical part of the background heat capacity has an interesting connection with some of the other amplitudes governing the heat capacity. Bagnuls and Bervillier have predicted $^{10}$ a universal amplitude ratio $R_{B_{\text {cr }}}^{ \pm}$defined by

$$
R_{B_{\mathrm{cr}}}^{ \pm} \equiv \frac{A^{ \pm}\left|D^{ \pm}\right|^{\alpha / \Delta_{\mathrm{I}}}}{\alpha B_{c}},
$$

with predicted values of $R_{B_{\mathrm{cr}}}^{+}=-0.70815$ for the ratio involving one-phase ${ }^{11}$ amplitudes $A$ and $D$, and $R_{B \text { cr }}^{-}$ $=-1.334 \pm 0.044$ for the two-phase ${ }^{6}$ region. One of their motivations was to allow an experimental test of the theory using fewer adjustable parameters. Others ${ }^{7,8,12}$ have noted that $R_{B_{\mathrm{cr}}}^{ \pm}$is not universal in the same sense as the universality of the critical exponents. Bagnuls and Bervillier ${ }^{13}$ have agreed, but noted that $R_{B_{\mathrm{cr}}}^{ \pm}$may be universal for a subclass of systems, and that the amplitude ratio $D^{+} / D^{-}$(predicted ${ }^{6}$ to be $0.96 \pm 0.25$ ) is similarly not universal.

Other amplitude ratios are predicted to be true universal quantities that should be the same for all systems in a given universality class (three-dimensional Ising for bulk, binary liquid mixtures). The ratio of the leading, singular, heat capacity amplitude in the one-phase region to that in the twophase region is now predicted to be $A^{+} / A^{-}=0.541 \pm 0.014$ by $\mathrm{RG}^{6}$ and $0.523 \pm 0.009$ by series ${ }^{9}$ calculation. One can also relate $A^{+}$to the amplitude $\xi_{0}$ of the correlation length in the one-phase region defined by $\xi=\xi_{0} t^{-\nu}$ using two-scale- factor universality: ${ }^{9} X=A^{+} \xi_{0}^{3} / k_{B}$, where $k_{B}$ is Boltzmann's constant, and $\nu$ is another universal critical exponent whose value $^{4}$ is about 0.63 . The value of $X$ is predicted from $\mathrm{RG}^{14}$ to be $X=0.01966 \pm 0.00017$ and from series ${ }^{9}$ to be 0.0188 \pm 0.00015 .

These predictions have been tested in other binary fluid mixtures with mixed results. ${ }^{1-3}$ Recent experiments measuring the heat capacity of binary fluid mixtures have given $A^{+} / A^{-}$in the range 0.56 to $0.71 ;^{15-18}$ a value consistently larger than the 0.52 value determined in liquid-vapor systems. ${ }^{19}$ To determine the universal quantity $X$, the value of the correlation length amplitude $\xi_{0}$ must be determined in a separate experiment. For the system triethylamine-water, the reported values of $\xi_{0}$ have varied from $0.10 \mathrm{~nm}$ (Ref. 15) to $0.125 \mathrm{~nm}$ (Ref. 20), with the most recent determination being $0.110 \pm 0.005 \mathrm{~nm}^{21}$ Since $\xi_{0}$ enters as the cube in $X$, the error in $\xi_{0}$ usually dominates the error in $X$. A number of values of $X$, ranging from 0.017 to 0.024 , have been determined using a variety of values for $\xi_{0}$ and $A^{+}$just for the system triethylamine and water. Recent determinations in other binary fluid systems have found $X$ to be $0.019 \pm 0.004$ in triethylamine and heavy water, ${ }^{17}$ and $0.020 \pm 0.002$ in 3-methylpentane and nitroethane. ${ }^{18}$

The value of $A^{+}$for the triethylamine-water system reported here has had a number of determinations using various techniques. Thoen et al. ${ }^{15}$ measured the heat capacity using a step technique in an adiabatic calorimeter which did not stir the sample. They found $A^{+}$to be $0.23 \pm 0.05$ $\mathrm{J} /\left(\mathrm{cm}^{3} \mathrm{~K}\right)$. Three other publications ${ }^{17,21,22}$ have reanalyzed those data and determined $A^{+}$to be between 0.23 and 0.36 $\mathrm{J} /\left(\mathrm{cm}^{3} \mathrm{~K}\right)$ (see Table I). One can also determine the amplitude $\mathrm{A}^{+}$from precise density measurements, along with the slope of the critical temperature dependence on pressure. Such determinations ${ }^{5,21,22}$ have given $A^{+}$between 0.119 and $0.35 \mathrm{~J} /\left(\mathrm{cm}^{3} \mathrm{~K}\right)$ as shown in Table I. Another possibility is to measure the refractive index and then calculate the density; however, the refractive index itself may have an intrinsic anomaly which is an issue not yet resolved. ${ }^{23,24}$ Values of $A^{+}$ determined by refractive index tend to be small, while values determined by density tend to be large, as is evident in Table I. One of the motivations of this experiment was to resolve this controversy by direct measurement.

\section{EXPERIMENT}

The heat capacity of a critical mixture of triethylamine and water was measured using a scanning adiabatic calorimeter similar to that used by Thoen et al. ${ }^{15,17}$ for studies in this same system and in triethylamine and heavy water. Our calorimeter improves upon Thoen's ${ }^{15}$ earlier experiment on this system by including stirring of the sample, operating in a scanning mode, and using computer data acquisition and control. An important advantage over ac calorimetry ${ }^{18}$ is our ability to measure the background heat capacity, which is important in theoretical constructions. A very slow temperature scan through the critical point is essential to allow the 
TABLE I. Amplitude of the specific heat anomaly in the one $\left(A^{+}\right)$and two $\left(A^{-}\right)$phase region for triethylamine-water $\left(\mathrm{TEA}-\mathrm{H}_{2} \mathrm{O}\right)$ as found by different techniques. The refractive index $n$ and density $\rho$ anomalies allow $A^{+}$to be calculated using thermodynamic relations. The amplitudes determined by analyzing prior heat capacity data (Ref. 15) depend on the choice of critical temperature and parameterization. A similar system, triethylamine-heavy water $\left(\right.$ TEA $\left.-\mathrm{D}_{2} \mathrm{O}\right)$, is provided for comparison. All errors are one standard deviation estimates as given by the authors.

\begin{tabular}{|c|c|c|}
\hline & $A^{+}\left(\mathrm{J} / \mathrm{cm}^{3} \mathrm{~K}\right)$ & $A^{-}\left(\mathrm{J} / \mathrm{cm}^{3} \mathrm{~K}\right)$ \\
\hline \multicolumn{3}{|l|}{$\begin{array}{l}\text { System/approach: } \\
\text { TEA- }-\mathrm{H}_{2} \mathrm{O}\end{array}$} \\
\hline 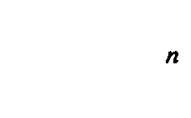 & $\left\{\begin{array}{l}0.116 \pm 0.005^{\mathrm{a}} \\
0.15 \pm 0.01^{\mathrm{b}} \\
0.271 \pm 0.007^{\mathrm{c}}\end{array}\right.$ & \\
\hline$\rho$ & $\left\{\begin{array}{l}0.119 \pm 0.009^{d} \\
0.35 \pm 0.02^{\mathrm{c}} \\
0.267 \pm 0.002^{\mathrm{c}}\end{array}\right.$ & \\
\hline$C_{p}$ & $\left\{\begin{array}{l}0.23 \pm 0.05^{\mathrm{f}} \\
0.23 \pm 0.02^{\mathrm{g}} \\
0.246 \pm 0.005^{\mathrm{h}} \\
0.36 \pm \mathbf{0 . 0 2 ^ { \prime }} \\
\mathbf{0 . 2 0 1} \pm \mathbf{0 . 0 0 6 ^ { \mathrm { k } }}\end{array}\right.$ & $0.358 \pm 0.005^{\mathrm{k}}$ \\
\hline \multicolumn{3}{|l|}{$\mathrm{TEA}-\mathrm{D}_{2} \mathrm{O}$} \\
\hline$C_{p}$ & $0.215 \pm 0.01^{j}$ & $0.378 \pm 0.004$ \\
\hline
\end{tabular}

"Reference 20 .

${ }^{\mathrm{b}}$ Reference 23.

${ }^{\mathrm{c}}$ Reference 21.

${ }^{\mathrm{d}}$ Reference 5.

'Reference 22

Reference 15

${ }^{8}$ Reanalysis of Ref. 15 in Ref. 17

heanalysis of Ref. 15 in Ref. 21

'Reanalysis of Ref. 15 in Ref. 22.

Keference 17.

${ }^{k}$ Our results in bold.

system to stay in quasistatic equilibrium throughout the experiment and thus considerable time and effort goes into the design and construction of the thermostat.

A critical composition sample of triethylamine and water was used. The triethylamine was $99 \%$ pure from Kodak and was further distilled over $\mathrm{KOH}$ with the center fraction saved under a dry nitrogen atmosphere in a Teflon bottle. The water was from E-M Science: "Omni-Solv glass distilled" which was frozen and vacuum pumped to remove dissolved gases before storage under nitrogen. The mixture used in this experiment was $31.7 \%$ by weight of triethylamine in water with a total mass of $16.015 \mathrm{~g}$ which is a volume of $17.20 \mathrm{~mL}$ at the critical point. This concentration of triethylamine and water is the most recently determined critical concentration ${ }^{25}$ and is close to the $32.1 \%$ used by other investigators of this system. ${ }^{5,15,17-18,20-24,26,27}$ The sample preparation was done under a nitrogen atmosphere and a small nitrogen and vapor bubble was left above the fluids to keep the pressure close to one atmosphere. This system exhibits a lower consolute point around $18.2^{\circ} \mathrm{C}$ and hence is one-phase below the critical point and two-phase above. Triethylamine is a strong base which is very reactive and requires special containment.

The mixture was sealed in a cylindrical, gold plated,

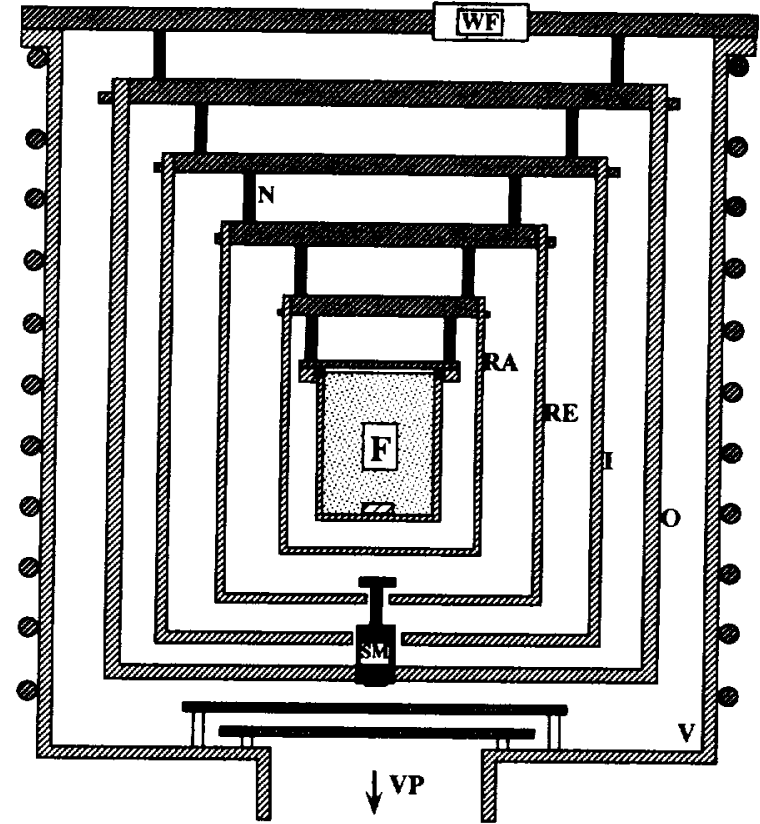

FIG. 1. Our adiabatic calorimeter is a set of temperature controlled, concentric cylindrical shells. The fluids $F$ are sealed in a gold-plated cell and are stirred by a stirbar coupled to a stir motor SM attached to the outer stage $O$. A passive radiation stage RA surrounds the cell and is contained within the reference stage $\mathrm{RE}$ which is actively temperature controlled to be at the same temperature as the cell. Stages are separated by nylon standoffs $N$. The inner stage $I$ and vacuum stage $V$ are also temperature controlled. A vacion pump VP reduces heat conduction losses by maintaining the pressure inside the thermostat to $10^{-6}$ tor while electrical signals pass through a wire feedthrough WF.

oxygen-free high conductivity copper cell with a Kalrez (DuPont perfluoroelastomer) o ring. The fluids were stirred with a Teflon coated stir bar which was magnetically coupled to a small motor attached to the outer stage of the thermostat as described below. Stirring the mixture is essential to achieve thermal equilibrium in the two-phase region. ${ }^{17,18,21,25}$ The fluids did not react with the cell as evidenced by a critical temperature which varied less than $2 \mathrm{mK}$ over a seven month period, which is in contrast to a $1 \mathrm{mK} /$ day drift when these fluids are sealed in glass. ${ }^{21,26}$ The cell is heated with a constant current (to $0.01 \%$ ) flowing through manganin wire wrapped in grooves on the outside of the cell body.

The cell is surrounded with a nested set of cylindrical, nickel-plated, copper stages forming a thermostat. A passive radiation shield surrounds the cell and is in turn encompassed by a reference stage that is actively temperature controlled so as to be at the same temperature as the cell throughout the experiment. Two additional actively controlled stages are maintained approximately $0.1 \mathrm{~K}$ below the next inner stage. The outermost of these has the stir motor attached to it. All the stages are attached to each other with hollow nylon stand-offs. This assembly is placed in a vacuum stage that has an ambient temperature determined by a Lauda RT-7 bath that circulates water through copper tubing soldered to the outside walls and lid, and is evacuated to 1 torr using a vacion pump. The thermostat is depicted in Fig. 1 . 
The temperature of the stages is determined by thermistors embedded through the lids and into the side wall of each stage. The cell and reference stage use matched, "ultrastable." Thermometrics thermistors while the other stages use Yellow Springs thermistors. All of the thermistors are wired in series with a $100 \mathrm{k} \Omega$ Vishay standard resistor and a $10 \mu \mathrm{A}$ constant current source. The resistance of each thermistor is determined by measuring the voltage across the standard resistor and across each thermistor using a $7 \frac{1}{2}$ digit Keithley 2001 multimeter. The power into the cell thermistor is about $2 \mu \mathrm{W}$ which is a negligible $0.05 \%$ of the power provided by the heater. This small power in the thermistor means that its self-heating is less than the temperature reproducibility of $0.2 \mathrm{mK}$. Each thermistor has been calibrated relative to a Guildline platinum resistance thermometer, which provides an accuracy of $10 \mathrm{mK}$ in absolute temperature and $0.3 \mathrm{mK}$ in relative temperature.

The digital multimeter measures the thermistor and standard resistor voltages and the voltage applied to the cell heater and reports each to a Macintosh IIci computer using a GPIB bus. The computer data acquisition and control software is written in the programming language LabVIEW and uses a software PID controlling algorithm to maintain the reference stage temperature within $1 \mathrm{mK}$ rms of the cell temperature. Two Kepco GPIB, digital to analog converters are used to set the heater voltages on the reference, inner, and outer stages. The program measures, controls and records temperatures with a cycle time of $12 \mathrm{~s}$. The cell heater receives $4 \mathrm{~mW}$ of power that causes the cell temperature to drift about $30 \mu \mathrm{K} / \mathrm{s}$ so that the cell temperature is effectively constant during each data cycle.

A subsequent calibration run using only water determined a number of experimentally important properties of the calorimeter. It was found that the power delivered to the fluids by the stirrer was less than $0.1 \%$ of the power supplied by the heater and thus negligible in this experiment. Using the known specific heat of water, we found that the empty cell contributes $20.4 \pm 0.1 \mathrm{~J} / \mathrm{K}$ to the total heat capacity. Small heat leaks between the cell and other stages were systematically quantified so corrections could be applied to the raw data on the critical mixture.

Two data runs are reported here on a critical composition of triethylamine and water between $14.5^{\circ} \mathrm{C}$ and $19.5^{\circ} \mathrm{C}$. Each data run takes almost two full days and entails over ten thousand sets of stage temperatures, cell temperature, and cell heater voltage. Early data runs gave a rounded anomaly and very noisy two-phase data as others ${ }^{17.18}$ have reported when stirring was not effective. The principle raw data are the variation in cell temperature with time, which can be analyzed in two ways to determine the amplitudes and exponents associated with the heat capacity. The first method is to determine the rate of change of the cell temperature $d T_{\text {cell }} / d t$ over some range of temperatures by doing a straight line, least-squares fit and then calculate the fluid's average heat capacity at the average temperature directly:

$$
C_{p}=\frac{\left[\frac{V^{2}}{R}-K_{i}\left(T_{\text {cell }}-T_{i}\right)\right]}{\left(d T_{\text {cell }} / d t\right) V_{0}}-1.19 \quad\left[\mathrm{~J} /\left(\mathrm{cm}^{3} \mathrm{~K}\right)\right],
$$

where $K_{i}$ is the thermal conductivity between the cell at temperature $T_{\text {cell }}$ and stage $i$ at temperature $T_{i}, V$ is the average, measured voltage across the cell heater of constant resistance $R, V_{0}$ is the fluid volume, and the empty cell heat capacity has been subtracted. The only significant heat loss was between the cell and reference stage for which we can correct using our calibration data on water. The number of points used to determine the cell temperature's rate of change needs to be large enough to minimize the effect of the temperature resolution yet not so large as to average over a region where the heat capacity changes significantly. We have chosen to use $200,100,50$, and 20 points to determine the slope $d T_{\text {cell }} / d t$ using the larger number of points furthest from the critical point. The heat capacities so determined from the two runs are listed in Table II and illustrated in Fig. 2. The errors represent the random fluctuations in the raw data and the propagated error from determining the slope of the cell temperature. It should be noted that the values of the heat capacity determined in this way are at a saturated vapor pressure, but Thoen et al. ${ }^{15}$ has demonstrated that the correction to constant pressure and composition is negligible and thus our values of the heat capacity are just $C_{p x}$

An alternative determination of the heat capacity parameters is to analyze the enthalpy as a function of the cell temperature. The enthalpy $H$ of a data run is calculated by adding the energy to the cell from the heater and subtracting the heat loss to other stages over each time interval $\Delta t$.

$$
H=\sum_{j}\left[\frac{V_{j}^{2}}{R}-K_{i}\left(T_{\text {cell }}-T_{i}\right)_{j}\right] \Delta t_{j} / V_{0} .
$$

The enthalpy is then related to the heat capacity by a simple integral over temperature $T$ :

$$
H=H_{0}+\int_{T_{0}}^{T} C_{p x} d T
$$

The calculated enthalpy divided by the critical temperature $T_{c}$, is illustrated in Fig. 3 for run 2. Using the enthalpy has the advantage of analyzing the entire data set, but the disadvantages of accumulated error (resulting in systematic offsets) and a $(1-\alpha)$ cusp instead of a divergence at the critical point. We will analyze our data using both techniques in the next section.

\section{ANALYSIS AND INTERPRETATION}

A weighted, nonlinear, least squares routine was used to fit the equations to the data by finding the best set of parameters that minimize ${ }^{28}$ the reduced chi square $\chi^{2} / N$. Using our own functions in the commercial program Igor Pro, ${ }^{29}$ we could allow some parameters to be free and force others to take on certain values as will be described below. This program allows each data point to be weighted according to the error in the dependent variable and determines errors on the parameters that are related to the diagonal elements of the 
TABLE II. Heat capacity data for two runs at the critical composition in triethylamine and water. Temperatures are in ${ }^{\circ} \mathrm{C}$ and the critical temperature is $18.225 \pm 0.001$ for run 1 and $18.227 \pm 0.001$ for run $2 . \delta C_{p}$ is the propagated error in $C_{p}$.

\begin{tabular}{|c|c|c|c|c|c|}
\hline Temperature & $\underset{\mathrm{J} /\left(\mathrm{cm}^{3} \mathrm{~K}\right)}{C_{p^{3}}}$ & $\underset{\mathrm{J} /\left(\mathrm{cm}^{\mathrm{\rho}} \mathrm{K}\right)}{\delta C}$ & Temperature & $\underset{\mathrm{J} /\left(\mathrm{cm}^{3} \mathrm{~K}\right)}{C_{p^{3}}}$ & $\underset{\mathrm{J} /\left(\mathrm{cm}^{3} \mathrm{~K}\right)}{\delta C_{p^{\prime}}}$ \\
\hline run 1 & & & un 2 & & \\
\hline 15.0121 & 4.08 & 0.01 & 15.1085 & 4.02 & 0.01 \\
\hline 15.2161 & 4.10 & 0.01 & 15.3136 & 4.02 & 0.01 \\
\hline 15.4293 & 4.13 & 0.01 & 15.5206 & 4.06 & 0.01 \\
\hline 15.6448 & 4.15 & 0.01 & 15.7263 & 4.07 & 0.01 \\
\hline 15.8616 & 4.18 & 0.01 & 15.9334 & 4.09 & 0.01 \\
\hline 16.0773 & 4.21 & 0.01 & 16.1414 & 4.12 & 0.01 \\
\hline 16.2945 & 4.23 & 0.01 & 16.3504 & 4.16 & 0.01 \\
\hline 16.5134 & 4.27 & 0.01 & 16.5514 & 4.21 & 0.01 \\
\hline 16.7328 & 4.32 & 0.01 & 16.729 & 4.25 & 0.01 \\
\hline 16.9527 & 4.37 & 0.01 & 16.904 & 4.30 & 0.01 \\
\hline 17.1148 & 4.42 & 0.02 & 17.0359 & 4.34 & 0.02 \\
\hline 17.2107 & 4.46 & 0.02 & 17.1222 & 4.37 & 0.02 \\
\hline 17.3052 & 4.48 & 0.02 & 17.2106 & 4.40 & 0.02 \\
\hline 17.3989 & 4.52 & 0.02 & 17.2984 & 4.44 & 0.02 \\
\hline 17.4922 & 4.57 & 0.02 & 17.3831 & 4.48 & 0.02 \\
\hline 17.5844 & 4.63 & 0.02 & 17.4695 & 4.57 & 0.02 \\
\hline 17.6762 & 4.69 & 0.02 & 17.5551 & 4.60 & 0.02 \\
\hline 17.7443 & 4.74 & 0.03 & 17.6389 & 4.63 & 0.02 \\
\hline 17.7878 & 4.78 & 0.03 & 17.7006 & 4.68 & 0.03 \\
\hline 17.8309 & 4.84 & 0.03 & 17.7434 & 4.71 & 0.03 \\
\hline 17.874 & 4.88 & 0.03 & 17.7851 & 4.76 & 0.03 \\
\hline 17.9143 & 4.94 & 0.03 & 17.8264 & 4.79 & 0.03 \\
\hline 17.9533 & 4.99 & 0.03 & 17.8668 & 4.86 & 0.03 \\
\hline 17.9919 & 5.06 & 0.03 & 17.9069 & 4.92 & 0.03 \\
\hline 18.0301 & 5.13 & 0.03 & 17.9479 & 4.97 & 0.03 \\
\hline 18.0678 & 5.24 & 0.03 & 17.9872 & 5.03 & 0.03 \\
\hline 18.1048 & 5.35 & 0.03 & 18.0254 & 5.12 & 0.03 \\
\hline 18.1411 & 5.51 & 0.03 & 18.063 & 5.21 & 0.03 \\
\hline 18.1763 & 5.76 & 0.03 & 18.1 & 5.34 & 0.03 \\
\hline 18.185 & 5.85 & 0.08 & 18.1363 & 5.50 & 0.04 \\
\hline 18.2018 & 6.05 & 0.08 & 18.173 & 5.77 & 0.04 \\
\hline 18.2177 & 6.80 & 0.09 & 18.182 & 5.88 & 0.08 \\
\hline 18.2222 & 7.32 & 0.23 & 18.2004 & 6.05 & 0.08 \\
\hline 18.2318 & 10.02 & 0.30 & 18.2177 & 6.58 & 0.08 \\
\hline 18.2361 & 9.56 & 0.29 & 18.2223 & 7.04 & 0.20 \\
\hline 18.2402 & 11.66 & 0.35 & 18.2319 & 10.53 & 0.31 \\
\hline 18.2444 & 10.03 & 0.30 & 18.2361 & 10.14 & 0.30 \\
\hline 18.2524 & 9.89 & 0.12 & 18.2402 & 11.36 & 0.34 \\
\hline 18.2644 & 9.90 & 0.12 & 18.2443 & 10.95 & 0.33 \\
\hline 18.2766 & 9.70 & 0.12 & 18.2484 & 10.59 & 0.32 \\
\hline 18.2891 & 9.25 & 0.12 & 18.2514 & 10.51 & 0.13 \\
\hline 18.3021 & 9.20 & 0.12 & 18.2619 & 9.98 & 0.13 \\
\hline 18.3086 & 9.03 & 0.06 & 18.2728 & 9.62 & 0.12 \\
\hline 18.3353 & 8.67 & 0.06 & 18.284 & 9.32 & 0.12 \\
\hline 18.3623 & 8.64 & 0.06 & 18.3014 & 9.01 & 0.06 \\
\hline 18.3895 & 8.47 & 0.06 & 18.3252 & 8.82 & 0.06 \\
\hline 18.418 & 8.29 & 0.06 & 18.3495 & 8.61 & 0.06 \\
\hline 18.4464 & 8.28 & 0.06 & 18.374 & 8.58 & 0.06 \\
\hline 18.4754 & 8.02 & 0.06 & 18.3989 & 8.36 & 0.06 \\
\hline 18.5047 & 8.01 & 0.06 & 18.4243 & 8.25 & 0.06 \\
\hline 18.5333 & 7.91 & 0.06 & 18.4497 & 8.10 & 0.05 \\
\hline 18.5614 & 7.83 & 0.06 & 18.4757 & 8.06 & 0.05 \\
\hline 18.589 & 7.74 & 0.06 & 18.5018 & 8.02 & 0.05 \\
\hline 18.616 & 7.72 & 0.05 & 18.528 & 7.95 & 0.05 \\
\hline 18.6431 & 7.64 & 0.05 & 18.5545 & 7.83 & 0.05 \\
\hline 18.7117 & 7.51 & 0.03 & 18.5812 & 7.78 & 0.05 \\
\hline 18.7673 & 7.44 & 0.03 & 18.5947 & 7.70 & 0.03 \\
\hline 18.8234 & 7.35 & 0.03 & 18.6491 & 7.58 & 0.03 \\
\hline 18.8799 & 7.30 & 0.03 & 18.7042 & 7.48 & 0.03 \\
\hline 18.9368 & 7.24 & 0.03 & 18.7602 & 7.35 & 0.03 \\
\hline 18.9942 & 7.18 & 0.03 & 18.8169 & 7.25 & 0.03 \\
\hline 19.0519 & 7.12 & 0.03 & $18.874 l$ & 7.19 & 0.03 \\
\hline
\end{tabular}


TABLE II. (Continued.)

\begin{tabular}{cccccc}
\hline \hline Temperature & $\begin{array}{c}C_{p} \\
\mathrm{~J} /\left(\mathrm{cm}^{3} \mathrm{~K}\right)\end{array}$ & $\begin{array}{c}\delta C_{p^{p}} \\
\mathrm{~J} /\left(\mathrm{cm}^{3} \mathrm{~K}\right)\end{array}$ & Temperature & $\begin{array}{c}C_{p_{p}} \\
\mathrm{~J} /\left(\mathrm{cm}^{3} \mathrm{~K}\right)\end{array}$ & $\begin{array}{c}\delta C_{p} \\
\mathrm{~J} /\left(\mathrm{cm}^{3} \mathrm{~K}\right)\end{array}$ \\
\hline 19.1107 & 7.10 & 0.03 & 18.9316 & 7.16 & 0.03 \\
19.1692 & 7.07 & 0.03 & 18.9892 & 7.13 & 0.03 \\
19.2277 & 7.02 & 0.03 & 19.0472 & 7.08 & 0.03 \\
19.2865 & 6.99 & 0.03 & 19.1055 & 7.03 & 0.03 \\
19.3456 & 6.96 & 0.03 & 19.1642 & 6.97 & 0.03 \\
19.4049 & 6.90 & 0.03 & 19.2232 & 6.94 & 0.03 \\
19.4645 & 6.89 & 0.03 & 19.2825 & 6.92 & 0.03 \\
& & & 19.3419 & 6.89 & 0.03 \\
& & & 19.4015 & 6.85 & 0.03 \\
& & & 19.4615 & 6.82 & 0.03 \\
\hline \hline
\end{tabular}

typical error matrix ${ }^{28}$ While this is adequate for equations whose parameters enter linearly, errors of nonlinear parameters should be determined by error ellipses. ${ }^{28}$ In tests on a subset of this data against a program ${ }^{30}$ that correctly determines the errors, we found that the parameter values were consistent, and that the Igor errors exceeded the appropriate, one standard deviation $(1 \sigma)$ errors. In the following, our quoted parameter errors are those given by the program and should be considered the $1 \sigma$ error.

In analyzing the heat capacity data shown in Table II, we use the equations

$$
\begin{aligned}
& C_{p}=C_{p_{0}}+E t+A|t|^{-\alpha}+D|t|^{\Delta_{1}-\alpha} \quad \text { (one phase), } \\
& C_{p}=C_{P_{0}}+E t+A^{\prime}|t|^{-\alpha}+D^{\prime}|t|^{\Delta_{1}-\alpha} \quad \text { (two phase), }
\end{aligned}
$$

where $t=\left(T-T_{c}\right) T_{c}$. In particular, we force equal exponent values above and below the critical point as predicted by theory. ${ }^{6}$ A smooth, continuous background specific heat near the critical point is expected, ${ }^{3,6}$ which means the background amplitudes $C_{p_{0}}$ and $E$ are the same in the one- and two-

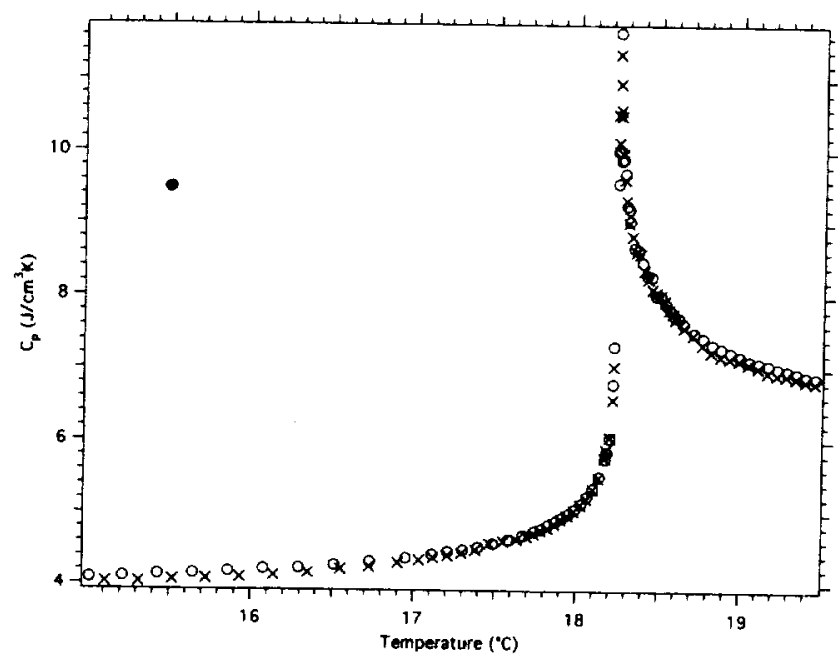

FIG. 2. The heat capacity $C_{p}$ of the fluid mixture triethylamine and water as given in Table II. Run $1(\mathrm{O})$ and run $2(\mathrm{X})$ are shown without their errors for clarity. A slight offset in the background heat capacity between the two runs is evident far from the critical point, which is at $T_{c}=18.226 \pm 0.002$. phase region. We simultaneously fit these equations to all the one- and two-phase data for each run. The exponent $\Delta_{1}$ is fixed at its theoretical value of 0.5 . This still leaves eight adjustable parameters: $C_{p_{0}}, E, A, A^{\prime}, D, D^{\prime}, T_{c}$, and $\alpha$. The parameter $E$ should reflect the noncritical fluid behavior and has a small negative value of $-4 \mathrm{~J} / \mathrm{K}$ for water in this temperature region which is effectively zero for the precision of our data. We do not find $E$ to improve the fits (see Table III) and hold its value at zero for most fits of the heat capacity data. Allowing the critical exponent $\alpha$ to vary gives a value of $0.10 \pm 0.01$, consistent with the theoretical value of 0.11 , but substantially increases the errors in the amplitudes. Thus, most fits have $\alpha$ fixed.

The two data runs reported in Table Il and shown in Fig. 2 have a small offset that is reflected in the value of $C_{p_{o}}$. The critical temperatures, as determined by the fits shown in Table III, were slightly larger (about $2 \mathrm{mK}$ ) for run 2 than run 1 but not significantly so. The two runs give a good sense of our reproducibility. Fits 1-1 through 1-5 allow various parameters to vary or be fixed at the values indicated. Allowing a correction to scaling term $\left(D, D^{\prime}\right.$ not zero) significantly improved the fit, but this form for our data does not allow a

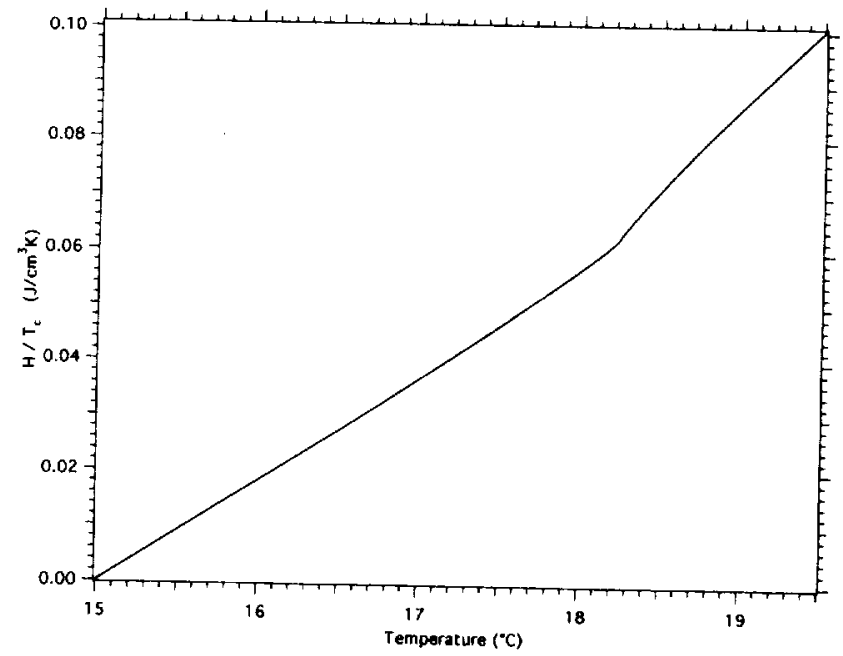

FIG. 3. A plot of the enthalpy $H$ values divided by the critical temperature

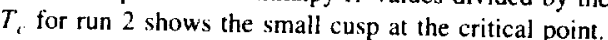


TABLE III. The heat capacity data from Table II are analyzed using Eq. (6) with $\Delta_{1}=0.5$. A nonlinear, weighted least-squares fit is simultaneously done in the one and wo phase region for each run. Units on $C_{p_{0}}, E, A, A^{\prime}, D$, and $D^{\prime}$, are $\mathrm{J} /\left(\mathrm{cm}^{3} \mathrm{~K}\right)$. The parameter errors are the one standard deviation uncertainty given by the fitting program. No error on a parameter means it was fixed. Values in brackets [ ] are calculated by imposing theoretical amplitude relations. The number before the dash in the "fit \#" indicates the run. An * marks the best fit in our judgment.

\begin{tabular}{|c|c|c|c|c|c|c|c|c|c|}
\hline Fit \# & $C_{p_{0}}$ & $E$ & $A$ & $A^{\prime}$ & $T_{r}$ & $D$ & $D^{\prime}$ & $\alpha$ & $x^{2} / N$ \\
\hline $1-1$ & $1.13 \pm 0.03$ & 0 & $1.78 \pm 0.02$ & $3.16 \pm 0.02$ & $18.2253 \pm 0.0006$ & 0 & 0 & 0.11 & 2.06 \\
\hline$* 1-2$ & $1.13 \pm 0.13$ & 0 & $1.78 \pm 0.05$ & $3.23 \pm 0.05$ & $18.2245 \pm 0.0006$ & $0.16 \pm 0.28$ & $-1.2 \pm 0.5$ & 0.11 & 1.65 \\
\hline $1-3$ & $0.97 \pm 0.05$ & $-6.2 \pm 1.3$ & $1.86 \pm 0.02$ & $3.26 \pm 0.02$ & $18.2253 \pm 0.0006$ & 0 & 0 & 0.11 & 1.72 \\
\hline $1-4$ & $0.4 \pm 0.7$ & 0 & $2.3 \pm 0.5$ & $4.0 \pm 0.6$ & $18.225 \pm 0.001$ & $0.7 \pm 0.5$ & $-1.9 \pm 0.8$ & $0.10 \pm 0.01$ & 1.61 \\
\hline $1-5$ & $1.14 \pm 0.26$ & $-4.8 \pm 2.4$ & $1.73 \pm 0.18$ & $3.10 \pm 0.23$ & $18.2251 \pm 0.0007$ & 0 & 0 & $0.114 \pm 0.006$ & 1.74 \\
\hline $1-6$ & $1.37 \pm 0.13$ & 0 & $1.69 \pm 0.05$ & $3.09 \pm 0.04$ & $18.2248 \pm 0.0007$ & $-0.05 \pm 0.3$ & {$[-0.9]$} & 0.11 & 2.05 \\
\hline $1-7$ & $1.92 \pm 0.02$ & 0 & $1.48 \pm 0.01$ & $2.90 \pm 0.01$ & $18.2238 \pm 0.0005$ & {$[-1.7]$} & {$[-2.8]$} & 0.11 & 2.53 \\
\hline $1-8$ & $1.62 \pm 0.01$ & 0 & $1.67 \pm 0.01$ & {$[3.08]$} & $18.2265 \pm 0.0007$ & {$[-2.0]$} & {$[-4.0]$} & 0.11 & 7.97 \\
\hline $2-1$ & $0.79 \pm 0.03$ & 0 & $1.94 \pm 0.02$ & $3.30 \pm 0.02$ & $18.2278 \pm 0.0006$ & 0 & 0 & 0.11 & 2.21 \\
\hline$* 2-2$ & $0.96 \pm 0.13$ & 0 & $1.87 \pm 0.05$ & $3.29 \pm 0.05$ & $18.2269 \pm 0.0007$ & $-0.2 \pm 0.3$ & $-1.5 \pm 0.5$ & 0.11 & 1.94 \\
\hline $2-3$ & $1.15 \pm 0.13$ & 0 & $1.80 \pm 0.05$ & $3.19 \pm 0.04$ & $18.2273 \pm 0.0007$ & $-0.8 \pm 0.2$ & {$[-1.3]$} & 0.11 & 2.13 \\
\hline $2-4$ & $1.69 \pm 0.02$ & 0 & $1.58 \pm 0.01$ & $3.01 \pm 0.01$ & $18.2262 \pm 0.0007$ & {$[-1.9]$} & {$[-3.4]$} & 0.11 & 2.41 \\
\hline $2-5$ & $1.46 \pm 0.01$ & 0 & $1.67 \pm 0.01$ & [3.08] & $18.2266 \pm 0.0007$ & $-1.4 \pm 0.1$ & {$[-2.5]$} & 0.11 & 2.19 \\
\hline
\end{tabular}

good determination of either $D$ or $D^{\prime}$. The best fit for run 1 is fit 1-2 (see Table III) not only because the reduced chi square is lowest, but the resulting equation is the simplest that fits the data. Fits 1-6 through 1-8 impose amplitude relations $6,10,11$ to reduce the number of variable parameters. Fit 1-6 allowed $A, A^{\prime}$, and $D$ to be free but calculated $D^{\prime}$ assuming $D^{+}=D^{-}$. The theoretical value of $R_{B_{\text {cr }}}^{ \pm}$was used to calculate $D$ and $D^{\prime}$ in fits 1-7 and 1-8 using the parameters $C_{p_{0}}, A$ and $A^{\prime}$. Fit 1-8 also imposes the theoretical constraint $A^{+} / A^{-}=A / A^{\prime}=0.541$, which allows one to fit the data with three parameters $\left(C_{p_{0}}, A, T_{c}\right)$ but gives a poor fit to the data. It should also be noted that the definition of $R_{B}^{ \pm}$ allows one to choose the sign of $D$ and we found negative values to give much better results. Bagnuls and Bervillier ${ }^{13}$ have argued that negative $D$ values are acceptable theoretically. None of these theoretical constraints designed to reduce the number of variable parameters provided a good fit of run 1 .

The fits to the second run of the heat capacity data are also shown in Table III. Since run 2 is very similar to run 1, the parameter values are quite consistent. Corresponding fits for the two runs are the pairs: $2-1$ and $1-1,2-2$ and $1-2$ (the best fits), 2-3 and 1-6, and 2-4 and 1-7. Fit 2-5 shows that a fairly good fit can result when $A / A^{\prime}$ is forced to be 0.541 and $D^{+} / D^{-}$is forced to be 1.0 . This fit can be used to calculate a value of $R_{B_{\mathrm{cr}}}^{+}=-0.63$, significantly less than the predicted $^{11}$ value of -0.71 .

Fits can also be done on the enthalpy, which is calculated by integrating the net power into the fluids over time as in Eq. (4). Using Eq. (5) with the expression for the heat capacity given in Eq. (6), we can express the enthalpy $H$ as

$$
\begin{aligned}
\frac{H}{T_{c}}= & \frac{H_{c}}{T_{c}}+C_{p_{0}} t+\frac{E}{2} t^{2}+\frac{A}{(1-\alpha)}|t|^{1-\alpha} \\
& +\frac{D}{\left(1+\Delta_{1}-\alpha\right)}|t|^{1+\Delta_{1}-\alpha} \quad \text { (one phase), }
\end{aligned}
$$

$$
\begin{aligned}
\frac{H}{T_{c}}= & \frac{H_{c}}{T_{c}}+C_{p_{0}} t+\frac{E}{2} t^{2}+\frac{A^{\prime}}{(1-\alpha)}|t|^{1-\alpha} \\
& +\frac{D^{\prime}}{\left(1+\Delta_{1}-\alpha\right)}|t|^{1+\Delta_{1}-\alpha} \quad \text { (two phase) }
\end{aligned}
$$

where $t$ is the reduced temperature, $H_{c}$ is the enthalpy at the critical point, and the other symbols are the same as in Eq. (6). The enthalpy has a cusp $(1-\alpha)$ anomaly at the critical point which is difficult to resolve when compared to the background (noncritical) quadratic dependence. Moreover, by integrating the raw data we sum any residual systematic errors in the experiment which appear as part of the "background" enthalpy. Since we take into account all known systematic errors in calculating the enthalpy, each data point is assigned the same error of $1 \times 10^{-5} \mathrm{~J} /\left(\mathrm{cm}^{3} \mathrm{~K}\right)$ in $H / T_{c}$. With the large number of data points (10274 for run $I$ and 11643 points for run 2), most of which are far from critical, the fits are inherently weighted to fit the region away from critical. Nevertheless, the resulting parameter values are quite consistent with those determined by the heat capacity fits (see Table IV).

In order to better determine the critical behavior, the range in temperature was restricted $\left(|t|<2 \times 10^{-3}\right)$ for some of the fits to the enthalpy for each run. By using enthalpy values close to the critical point, the correction to scaling terms $D$ and $D^{\prime}$ should be zero while the cusp anomaly with amplitudes $A$ and $A^{\prime}$ should be significant. The exponent $\alpha$ was free in some of the fits and its value was determined to be $0.107 \pm 0.006$, which is quite consistent with theoretical predictions $^{9}$ of $0.110 \pm 0.003$. We fix the value of $\alpha$ for most of the fits so that the amplitudes, which are sensitive to the exponent value, can be compared to other experimental and theoretical determinations where $\alpha$ was set at 0.11 . The reduced range fits $1-D$ and $2-D$ in Table IV show that the amplitudes $A$ and $A^{\prime}$ are unchanged from the full range fits $1-B$ and $2-A$ in which correction to scaling terms are included. Moreover, the reduced range fits $1-E$ and $2-F$ show 
TABLE IV. The enthalpy $H$, as calculated by Eq. (4) and in units of $\mathrm{J} / \mathrm{cm}^{3}$, is fitted by Eq. (7). The parameters and notations are defined in Table III. The Cor run 1 and 18.227 for run 2 .

\begin{tabular}{|c|c|c|c|c|c|c|c|c|c|c|}
\hline Fit \# & range & $10^{2} H_{c} / T_{c}$ & $C_{p_{0}}$ & $E$ & $A$ & $A^{\prime}$ & $\alpha$ & $D$ & $D^{\prime}$ & $\chi^{2} / N$ \\
\hline $1-A$ & full & $5.544 \pm 0.001$ & $1.19 \pm 0.01$ & 0 & $1.787 \pm 0.002$ & $3.179 \pm 0.002$ & 0.11 & 0 & 0 & 4.4 \\
\hline$* 1-B$ & full & $5.538 \pm 0.001$ & $1.53 \pm 0.01$ & 0 & $1.634 \pm 0.006$ & $3.105 \pm 0.006$ & 0.11 & $-0.55 \pm 0.03$ & $-2.33 \pm 0.07$ & 2.04 \\
\hline $1-C$ & full & $5.541 \pm 0.001$ & $1.45 \pm 0.12$ & $-19 \pm 1$ & $1.84 \pm 0.09$ & $3.39 \pm 0.12$ & $0.101 \pm 0.002$ & $-2.4 \pm 0.2$ & $-3.0 \pm 0.1$ & 1.67 \\
\hline $1-D$ & $|t|<2 \times 10^{-3}$ & $5.541 \pm 0.001$ & $1.42 \pm 0.01$ & 0 & $1.674 \pm 0.006$ & $3.093 \pm 0.006$ & 0.11 & 0 & 0 & 4.15 \\
\hline $1-E$ & $|t|<2 \times 10^{-3}$ & $5.538 \pm 0.001$ & $1.45 \pm 0.01$ & $-54 \pm 1$ & $1.634 \pm 0.006$ & $3.105 \pm 0.006$ & 0.11 & 0 & 0 & 2.95 \\
\hline${ }^{*} 2-A$ & full & $6.215 \pm 0.001$ & $1.09 \pm 0.01$ & 0 & $1.838 \pm 0.002$ & $3.267 \pm 0.006$ & 0.11 & $-0.46 \pm 0.03$ & $-1.81 \pm 0.07$ & 2.31 \\
\hline $2-B$ & full & $6.215 \pm 0.001$ & $0.97 \pm 0.03$ & 0 & $1.94 \pm 0.02$ & $3.40 \pm 0.03$ & $0.107 \pm 0.001$ & $-0.4 \pm 0.1$ & $-2.0 \pm 0.1$ & 2.27 \\
\hline $2-C$ & full & $6.216 \pm 0.001$ & $1.12 \pm 0.04$ & $-11.8 \pm 0.1$ & $1.94 \pm 0.03$ & $3.41 \pm 0.03$ & $0.105 \pm 0.001$ & $-1.9 \pm 0.1$ & $-2.4 \pm 0.1$ & 1.95 \\
\hline $2-D$ & $|t|<2 \times 10^{-3}$ & $6.215 \pm 0.001$ & $1.16 \pm 0.01$ & 0 & $1.797 \pm 0.006$ & $3.203 \pm 0.006$ & 0.11 & 0 & 0 & 2.00 \\
\hline $2-E$ & $|t|<2 \times 10^{-3}$ & $6.215 \pm 0.001$ & $1.31 \pm 0.03$ & 0 & $1.69 \pm 0.02$ & $3.06 \pm 0.02$ & $0.113 \pm 0.002$ & 0 & 0 & 1.97 \\
\hline $2-F$ & $|t|<2 \times 10^{-3}$ & $6.214 \pm 0.001$ & $1.15 \pm 0.01$ & $-26.7 \pm 0.2$ & $1.791 \pm 0.006$ & $3.215 \pm 0.006$ & 0.11 & 0 & 0 & 1.69 \\
\hline
\end{tabular}

that including $E \neq 0$ improves the fit but does not change the values of the amplitudes $A$ and $A^{\prime}$. The same is not the case for the full range fits $1-C$ and $2-C$ where allowing $E \neq 0$ causes the amplitudes $A$ and $A^{\prime}$ to increase. Therefore, we are more confident in the consistent values of $A$ and $A^{\prime}$ determined when $E=0$. The enthalpy data require the use of extended scaling for the entire range $\left(|t|<10^{-2}\right)$ but the values depend heavily on the whether $E$ is zero or free (compare fits $1-B$ to $1-C$ and $2-A$ to $2-C$ ). Because of the consistency of $A$ and $A^{\prime}$ in the restricted and full range fits, we favor the values of $D$ and $D^{\prime}$ as determined in fits $1-B$ and 2-A, which is why we label these as our best fits over the entire range.

The heat capacity fits given in Table III and the enthalpy fits in Table IV provide a consistent set of amplitude values when $E=0$ and $\alpha=0.11$, which can be converted to the amplitudes of Eq. (1) to be: $B_{C}=-2.7 \pm 0.2, A^{+}=0.201 \pm 0.006$, $A^{-}=0.358 \pm 0.005 \quad \mathrm{~J} /\left(\mathrm{cm}^{3} \mathrm{~K}\right), \quad D^{+}=-0.29 \pm 0.04, \quad$ and $D^{-}=-0.65 \pm 0.10$, where all the errors are one standard deviation estimates. This value of $A^{+}$is somewhat larger, while the value of $A^{-}$is somewhat smaller, than Thoen et al. ${ }^{15}$ found in their heat capacity experiment, yet both are within experimental error of those earlier values (see Table I). However, $A^{+}$is inconsistent with all the values determined using either density or refractive index measurements, whose values tend to be inconsistent with each other. Our value of the universal ratio $A^{+} / A^{-}=0.56 \pm 0.02$ was quite consistent with experimental values determined in several binary-liquid systems recently (see Table V) and slightly larger than theoreti-

TABLE V. Amplitude ratios determined from this experiment. Various techniques have been used to determine $A^{+}$in TEA-water (values in Table I) and determine $X$ using assorted values of $\xi_{0}$; our determination uses the most recently measured value. Three other recently measured binary liquid systems (triethylamine-heavy water $\left[\mathrm{TEA}-\mathrm{D}_{2} \mathrm{O}\right], 3-$-methylpentane + nitroethane $[3 \mathrm{MP}-\mathrm{NE}]$, and 2,6-lutidine + water $\left[2,6 \mathrm{~L}-\mathrm{H}_{2} \mathrm{O}\right]$ ) are provided for in $\mathrm{SF}_{6}$ are also given.

\begin{tabular}{|c|c|c|c|c|c|}
\hline System/technique & $A^{+} / A^{-}$ & $D^{+} / D^{-}$ & $X$ & $R_{B_{c r}}^{+}$ & $R_{B_{\text {cr }}}^{-}$ \\
\hline \multicolumn{6}{|l|}{$\mathrm{TEA}-\mathrm{H}_{2} \mathrm{O}$} \\
\hline$\rho$ & $0.36 \pm 0.03^{\mathrm{a}}$ & & $0.017 \pm 0.001^{\mathrm{a}}$ & & \\
\hline$n$ & & & $0.018 \pm 0.002^{\mathrm{a}}$ & & \\
\hline$C_{p}$ & $0.7 \mathrm{I} \pm 0.20^{\mathrm{b}}$ & & $0.017 \pm 0.006^{b}$ & & \\
\hline$n, \rho$ & & & $0.024 \pm 0.007^{c}$ & & \\
\hline $\mathrm{C}_{\mathrm{p}}$ & $0.56 \pm 0.02^{d}$ & $0.5 \pm 0.1^{d}$ & $0.019 \pm 0.003^{d}$ & $-0.5 \pm 0.1^{d}$ & $-1.1 \pm 0.1^{d}$ \\
\hline $\mathrm{TEA}-\mathrm{D}_{2} \mathrm{O}$ & $0.57 \pm 0.01^{e}$ & $-0.5^{\mathrm{c}}$ & $0.019 \pm 0.004^{e}$ & $-0.48 \pm 0.01^{f}$ & $-0.96 \pm 0.03^{f}$ \\
\hline $\begin{array}{l}3 \mathrm{MP}-\mathrm{NE} \\
2,6 \mathrm{~L}-\mathrm{H}_{2} \mathrm{O}\end{array}$ & $\begin{array}{l}0.56 \pm 0.02^{\mathrm{g}} \\
0.57 \pm 0.25^{\mathrm{h}}\end{array}$ & $1.0 \pm 0.4^{8}$ & $0.020 \pm 0.002^{8}$ & & \\
\hline Liquid-vapor & $0.52 \pm 0.02^{i}$ & $1.4^{j}$ & & & \\
\hline \multicolumn{4}{|l|}{ Theory } & & \\
\hline $\mathbf{R G}$ & $0.541 \pm 0.014^{m}$ & $0.96 \pm 0.25^{\mathrm{m}}$ & $0.0197 \pm 0.0002^{n}$ & $-0.70815^{p}$ & $-1.334 \pm 0.044^{m}$ \\
\hline $\begin{array}{l}{ }^{\mathrm{a}} \text { Reference } 27 . \\
{ }^{\mathrm{b}} \text { Reference } 15 . \\
{ }^{\mathrm{C}} \text { Reference } 21 . \\
{ }^{\mathrm{d}} \text { Our results in bold. } \\
\text { 'Reference } 17 . \\
\text { 'Calculated from par } \\
\text { in Table III of Ref } \\
\text { 'Reference } 18 .\end{array}$ & $\begin{array}{l}\text { rameter values } \\
\text { f. } 17 \text {. }\end{array}$ & & $\begin{array}{l}{ }^{{ }^{h}} \text { Reference } \\
\text { 'Reference } \\
{ }^{j} \text { Reference } \\
{ }^{{ }^{k}} \text { Reference } \\
{ }^{m_{R}} \text { Referenc } \\
{ }^{{ }^{m}} \text { Reference } \\
{ }^{\mathrm{P}} \text { Reference }\end{array}$ & $\begin{array}{l}16 . \\
19 . \\
2 . \\
9 . \\
6 . \\
14 . \\
11 .\end{array}$ & \\
\hline
\end{tabular}


cal predictions using either renormalization group ${ }^{6}$ or series ${ }^{9}$ calculations. The amplitude ratio $D^{+} / D^{-}=0.5 \pm 0.1$ is no longer thought to be universal ${ }^{1,3}$ but is expected to be similar in similar systems. The value we find is the same magnitude, but opposite sign, of that found in the triethylamine-heavy water system. ${ }^{17}$ and about one half the value found in 3 -methylpentane + nitroethane 18 and predicted by theory. "Fit 2-5 indicated that our data are not inconsistent with $D^{+} / D^{-}=1$, but these difficult to determine, correction to scaling amplitudes are best determined in our enthalpy fits, which favor a lower ratio.

Two other universal amplitude ratios can be determined from our experiment. The two-scale-factor universality ratio $X=A^{\prime} \xi_{0}^{3} / k_{B}$ can be calculated from our value of $A^{+}$and the most recently published ${ }^{21}$ value for $\xi_{0}=0.110 \pm 0.005 \mathrm{~nm}$, which gives $X=0.019 \pm 0.003$. This value agrees with other recent experimental determinations and with theoretical predictions (see Table $V$ ).

It is not certain whether another amplitude ratio $R_{B}^{+}$ given by Eq. (2) is universal, 7.8 .12 .13 however, theoretical predictions have been made ${ }^{6,11}$ which have not previously been tested in a binary fluid mixture. Even though we could not determine $D$ with much precision, it enters into this amplitude ratio raised to a small power and thus is not so crucial. We find $R_{B_{\mathrm{cr}}}^{r}=-0.5 \pm 0.1$ and $R_{B_{\mathrm{cr}}}^{-}=-1.1 \pm 0.1$, which are both smaller than predicted (see Table $V$ ). Using the fit parameters Bloemen ef al. ${ }^{17}$ determined for the system triethylamine and heavy water, we can calculate values for $R_{B_{\text {ir }}}$ which are consistent with ours (see Table V). Belyakov and Kiselev ${ }^{x}$ feel that it would be difficult to distinguish the singular specific heat $B$, from the regular part and thus making an experimental check of the universality of $R_{B_{\text {or }}}^{+}$"probably impossible." The system studied here has the advantage of a constant specific heat in the one-phase region far from the critical temperature $\left[B_{h s}=4.07 \mathrm{~J} /\left(\mathrm{cm}^{3} \mathrm{~K}\right)\right.$ in run 1 and 4.01 $\mathrm{J} /\left(\mathrm{cm}^{3} \mathrm{~K}\right)$ in run 2], which together with the fitted $C_{P_{0}}$ allows a good approximation to $B_{\text {. }}$. It is thus possible to begin the process of experimental determination of a possibly universal value for $R_{H_{\text {ir }}}$

\section{CONCLUSION}

The heat capacity of the binary liquid mixture triethylamine-water has been measured near its lower critical consolute point using a scanning, adiabatic calorimeter. The critical exponent $\alpha$ was determined to be $0.107 \pm 0.006$, consistent with theoretical predictions. While our values of $A^{+}=0.201 \pm 0.006$ and $A=0.358 \pm 0.005 \mathrm{~J} /\left(\mathrm{cm}^{3} \mathrm{~K}\right)$ for $\alpha=0.11$ were consistent with a previous heal capacity measurement. ${ }^{15}$ the value of $A$ " was inconsistent with values determined by density or refractive index measurements $5.20-23$ in this triethylamine and water system. Jacobs and Greer $^{31}$ have reviewed the values of $A$ as ohtained from density measurements and find them not consistent with those obtained from $C_{p}$ measurements: they suggest that the discrepancy is due to ignoring the behavior of $C_{t}$ near $T_{1}, 2$
Various amplitude ratios were calculated from the best fits to our data with $\alpha=0.11: A^{+} / A^{-}=0.56 \pm 0.02$. $D^{*} / D^{--}=0.5 \pm 0.1, X=0.019 \pm 0.003, R_{B_{\mathrm{cr}}}^{+}=-0.5 \pm 0.1$, and $R_{R_{\mathrm{u}}}^{-}=-1.1 \pm 0.1$. While $A^{+} / A^{-}$was consistent with other recent experimental determinations in binary liquid mixtures, it was slightly larger than both theoretical predictions and recent experimental determinations in the simple liquid-gas systems $\mathrm{CO}_{2}$ and $\mathrm{SF}_{6}{ }^{19}$ The correction to scaling amplitude ratio $D^{+} / D^{-}$was half of that predicted, but experimental values are still quite scattered. As a result of more precise theoretical calculations and experimental determinations, the two-scale-factor universality ratio $X$ now is consistent among experiments and theories. We could not reduce the number of variable parameters in our fits by using $R_{B_{\mathrm{cr}}}^{+}$, but that is probably because its universal value has not been determined. Our determination of $R_{B_{\text {cr }}}^{\ddagger}$ is smaller than predicted and is the first such determination in a binary fluid mixture.

\section{ACKNOWLEDGMENTS}

The authors are indebted to Ali Özgenç for calibrating the calorimeter using water and to numerous students who have made this research possible. We are also grateful for helpful suggestions by S. C. Greer and M. A. Anisimov. This research was supported by NASA Grant No. NAG3-1404 with early support coming from NSF Grant No. CHE8722034. A.C.F. acknowledges summer support from NSF Grant No. DMR-9.322301.

\footnotetext{
'J. V. Sengers and J. M. H. Levelt-Sengers, Annu. Rev. Phys. Chem. 37. $189(1986)$

'S. C. Greer and M. R. Moldover, Annu. Rev. Phys. Chem. 32. 233 (1981).

${ }^{3}$ A. Kumar. H. R. Krishnamurthy, and E. S. R. Gopal, Phys. Rep. 98, 57 (1983).

'M. F. Fisher and J.-H. Chen, J. Phys. (Paris) 46. 1645 (1985)

${ }^{5}$ D. Beysens, A. Bourgou, and P. Calmettes, Phys. Rev, A 26, 3589 (1982).

"C. Bitgnuls, C. Berviltier, D. I. Meiron, and B. G. Nickel, Phys. Rev. B 35 , $3.58511987)$.

M. A. Anisimov, E. E. Gorodetskii, V. D. Kulikov, and J. V. Sengers. Phys. Rev, E 51, 1199 (1995): M. A. Anisimov, S. B. Kiselev, J. V. Sengers, and S. Tang, Physica A 188, 487 (1992).

M. Y. Belyakov and S. B. Kiselev. Physica A 190. 75 (1992).

"A. J. Liu and M. E. Fisher, Physica A 156, 35 (1989).

"C. Bagnuls and C. Bervillier. Phys. Lett. 107A. 299 (1985): 112A. 477 119851

"C. Bagnuls and C. Bervillier. Phys. Rev. B 32, 7204 (1985).

I: J. F. Nicoll and P. C. Albright, Phys. Rev. B 34. 1991 (1986).

${ }^{1} \mathrm{C}$. Bagnuls and C. Bervillier. Phys. Lett. 195A, 16.3 (1994).

${ }^{14} \mathrm{C}$. Bervillier and C. Godreche, Phys. Rev. B 21, $5427(1980)$.

${ }^{15} \mathrm{~J}$. Thoen. E. Bloemen, and W. Van Dael, J. Chem. Phys, 68, 735 (1978)

${ }^{\text {it }}$ L. V. Entow. V. A. Levehenko, V. P. Voronov. Int. J. Thermophys. 14. $221(1903)$

"E. Blocmen. J. Thoen. and W. Van Dael, J. Chem. Phys. 73, 4629 (1980).

"G. Sanches. M. Meichle. and C. W. Garland. Phys. Rev. A 28. 1647 119831

"W. Stauth. A. Haupt, and I. Eicher, Int. J. Thermophys. 16, 103.3 (1995) and references contatined therein.

"D. Beysens, R. Tufieu. and Y. Garrabos, J. Phys Lell. (Paris) 40,623 (10)701)

2" (i. Kalczer and D). Beysens. J Chem. Phys 92,6747 (1990).

$\because$ G. P. Jurrow and S. C. Cireer. J Chem. Phys. 79. 3474119831

'C. Pepin. T. K. Bose, and J. Thoen, Phys Rev. lett 60. 2507 (1988).
} 
${ }^{24}$ D. Beysens and G. Zalczer, Phys. Rev. Lett. 65, 1690 (1990); C. Pépin, T. K. Bose, and J. Thoen, ibid. 65, 1691 (1990); D. Beysens and G. Zalczer, Europhys. Lett. 8, 777 (1989).

${ }^{25}$ R. Gastaud, D. Beysens, and G. Zalczer, J. Chem. Phys. 93, 3432 (1990).

${ }^{26}$ H. Chaar, M. R. Moldover, and J. W. Schmidt, J. Chem. Phys. 85, 418 (1986).

${ }^{27}$ D. Beysens and A. Bourgou, Phys. Rev. A 19, 2407 (1979).
${ }^{28}$ P. R. Bevington, Data Reduction and Error Analysis for the Physical Sciences (McGraw-Hill, New York, 1969).

${ }^{29}$ WaveMetrics, Inc., Lake Oswego, OR 97035

${ }^{30} \mathrm{~K}$. A. Andrews and D. T. Jacobs, "Fitafn4.tru a weighted, nonlinear, least-squares fitting routine in True Basic" (unpublished)

"1'D. T. Jacobs and S. C. Greer (unpublished).

${ }^{32}$ G. Morrison and C. M. Knobler, J. Chem. Phys. 65, 5507 (1976). 